\title{
Far sidelobes measurement of the Atacama Cosmology Telescope
}

\author{
Rolando Dünner ${ }^{a, b}$, Patricio Gallardo ${ }^{a, b}$, Ed Wollack ${ }^{c}$, Fernando Henriquez ${ }^{d}$ and Carlos \\ Jerez-Hanckes $^{d}$ \\ ${ }^{b}$ Departamento de Astronomía, Facultad de Física, Pontificia Universidad Católica de Chile, \\ Vicuña. Mackenna 4860, Santiago, Chile \\ ${ }^{a}$ Centro de Astro Ingeniería, Pontificia Universidad Católica de Chile, Vicuña Mackenna 4860, \\ Santiago, Chile \\ ${ }^{c}$ Code 553/665, NASA/Goddard Space Flight Center, Greenbelt, MD 20771, USA

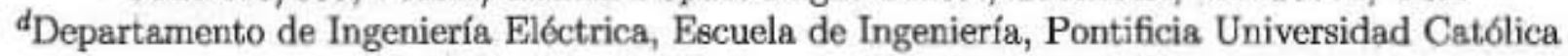 \\ de Chile, Vicuña Mackenna 4860, Santiago, Chile
}

\begin{abstract}
The Atacama Cosmology Telescope (ACT) is a $6 \mathrm{~m}$ telescope designed to map the Cosmic Microwave Background (CMB) simultaneously at $145 \mathrm{GHz}, 220 \mathrm{GHz}$ and $280 \mathrm{GHz}$. Its off-axis Gregorian design is intended to minimize and control the off-axis sidelobe response, which is critical for scientific purposes. The expected sidelobe level for this kind of design is less than $-50 \mathrm{~dB}$ and can be challenging to measure. Here we present a measurement of the $145 \mathrm{GHz}$ far sidelobes of ACT done on the near-field of the telescope. We used a $1 \mathrm{~mW}$ microwave source placed 13 meters away from the telescope and a chopper wheel to produce a varying signal that could be detected by the camera for different orientations of the telescope. The source feed was designed to produce a wide beam profile. Given that the coupling is expected to be dominated by diffraction over the telescope shielding structure, when combined with a measurements of the main beam far fiela response, these measurement can be used to validate elements of optical design and constrain the level of spurious coupling at large angles. Our results show that the diffractive coupling beyond the ground screen is consistently below $-75 \mathrm{~dB}$, satisfying the design expectations.
\end{abstract}

Keywords: ANTENNAS, SIDELOBES, SUB-MM ASTRONONY, COSMIC MICROWAVE BACKGROUND

\section{INTRODUCTION}

The Atacama Cosmology Telescope (ACT) is a 6-meter dish millimeter wave telescope located at an elevation of $5200 \mathrm{~m}$ at Cerro Toco in northern Chile. It is designed to measure the Cosmic Microwave Background (CMB) redlation producing high sensitivity maps with arcrninute resolution, sampling multipoles up to $\ell \approx 10^{4}{ }^{1}$ Between years 2007 and 2011, ACT was equipped with MIBAC, a camera which observed simuitaneously in three bands centered at $145 \mathrm{GHz}, 220 \mathrm{GHz}$ and $280 \mathrm{GHz}$, with bandwidths of epproximately $30 \mathrm{GHz}_{\mathrm{z}}$ for each frequency, and each one sampled with a kilopixel TES bolometer array. ${ }^{2}$ These frequency bands were chosen to sample the decrement, null and increment regions of the thermal Sunyaev-Zel'dovich effect in galoxy chnsters, ${ }^{3}$ permitting their direct detection from the maps. ${ }^{4,5}$ The observation strategy consisted in scanning the sky in aximuth at a fixed elevation, sampling a zig-zag on the sky while the Earth rotates. This technique guarantees that the airmass stays constant, the cryogenics are stable, the telescope deformations are small and that the local environment is sampled in a consistent way. During the mentioned years of operation, ACT observed two long stripes of the sky, one centered at declination $-51 \mathrm{deg}$ and the other at the equator, covering nearly 2000 square degrees in total. A long list of scientific and technical publications have come out from these studies. ${ }^{4-8}$

The off-axis Gregorian design of the telescope is intended to minimize and control the off-axis sidelobe response, which is critical for scientific purposes. Moreover, in order to minimize the optical contamination coming from the ground and the surroundings, a shielding structure was buit around the optical path between

R.D.: E-mail: rdunnerQastro.puc.cl; P.G.: E-mail: pngallaraing-puc.cl 
the primary and secondary mirrors, and another ground shield was built around the whole telescope to complete the isolation of the system. The expected sidelobe level for this kind of design is less than $-50 \mathrm{~dB}$ and can be challenging to measure. To validate the design and support the scientific results, experiments to characterize the far sidelobes were performed.

Here we present the method and results of an experiment done on the ACT telescope to measure its far sidelobes for the $145 \mathrm{GHz}$ band. In Section 2 we discuss basic antenna response concepts, in Section 3 we describe the experimental method that was used, in Section 4 we explain the techniques used to analyze the data, in Section 5 we present our results, and in Section 6 we give our conclusions about the experiment.

\section{ANTENNA PROFILE AND SIDELOBES}

The response of a radio telescope to a single point source (plane wave) is given by its normalized antennn power pattern (also known as normalized point spread function or PSF), defined as

$$
\int_{6 *} P(\theta, \phi) d \Omega=1 .
$$

Then, the observed antenna temperature $T_{a}$ results from the couvolution of the PSF with the sky brightness temperature $T_{b}$ :

$$
T_{a}(\theta, \phi)=\int_{4 \pi} T_{b}\left(\theta^{\prime}, \phi^{\prime}\right) P\left(\theta^{\prime}-\theta, \phi^{\prime}-\phi\right) d \Omega^{\prime} .
$$

For a single dish teiescope, the anterna pattern consists in a central beam (at $\theta=\phi=0$ ) followed by several sidelobes of lower intensity at increasing angles from the center. The width of the central beam is usually defined by the full width half maximum (FWHM) or the angular size of the $-3 \mathrm{~dB}$ contour of the antenna pattern. The FWHM is limited by diffraction at the antenna aperture. For a circular aperture of diameter $D$ it is expected to be

$$
\theta_{\text {rwhat }}=1.02 \frac{\lambda}{D}[\mathrm{rad}],
$$

where $\lambda$ is the radiation wavelength.

The remaining local maxima of the antenns pattern are called sidelobes. There can be near and far sidelobes depending on their angular distance from the main beam, where "near" usually means a few beam sizes away from the center. We can see that the total antenna temperature will be formed by the contribution from the main beam and the sidelobes. Writing this explicitly we get

$$
T_{a}(\theta, \phi)=\left(\int_{m b}+\int_{n s l}+\int_{f a l}\right) T_{b}\left(\theta^{\prime}, \phi^{\prime}\right) P\left(\theta^{\prime}-\theta, \phi^{\prime}-\phi\right) d \Omega^{\prime},
$$

where $m b$ is the main bearn, $n s l$ is the near sidelobe region and $f s l$ is the far sideiobe region. Depending on the magnitude of the sidejobes, they can constitute s significant distortion to the measurements, especially when making maps of extended areas of the sky or when observing extended sources.

Near sidelobes are usually originated by the geometrical properties of the optical design (including illumination pattern and blockage), as well as by defects in the setup like surface errors, reflector misalignment and defocus. For example, they appen: naturally from the Bessel functions describing the PSF solution of a circular aperture. They are relatively strong, reaching between $-30 \mathrm{~dB}$ and $-17 \mathrm{~dB}$, and can sometimes be measured directly using strong astronomical point sources. ${ }^{\circ}$ They can also be modeled using theory of diffraction and several commercial software packages are useful in this regime. Finally, as they appear close to sources within the same map, it is at least plausible to deconvolve their effects form the scientific results.

Far sidelobes instead are harder to charecterize. They are usually caused by diffraction, reflection or blockage from macroscopic parts of the telescope, as well as direct spillover from the camera. They are normally broad and very weak (of the order of $-50 \mathrm{~dB}$ or lower), being generally more difficult to measure. ${ }^{9} \mathrm{On}$ top of this, they are also harder to simulate, as now the simulation must include parts of the telescope outside the optical path and of physical scales much larger than the wavelength (of the order of the size of the teleacope), which highly complicates 
the tesk of finite element or boundary solvers, becoming computationally expensive. Mloreover, far sidelobes are usually excited by strong sources outside the mapping region, making their effects on the science much harder to understand and treat. For example, the ground in the surroundings of the telescope can couple through the far sidelobes and contribute significantly to the antenna temperature given its high brightness temperature and its large anguiar size. ${ }^{9}$ The far sidelobes must be considered, minimized, and ultimately controlled in the initial telescope design to enable reliable scientific results for continuum mapping experiments.

\section{METHOD}

In the case of ACT, we expect the far sidelobe contribution to be produced by diffraction from the edges of the shielding structure and reflection from features near the optical path. For this reason, our method consisted in illuminating the telescope with a $145 \mathrm{GHz}$ source placed on the edge of the external ground shield and measuring the power received from different orientations of the telescope. Given that diffracted light would shine in basically all directions, we required the source to have a very wide antenna pattern such that it would illuminate the whole telescope structure even from a close location. The signal was modulated with a chopper wheel in front of the feec to modulate it away from the $1 / f$ noise of the system. By carefully characterizing power emitted by the source, we are able estimate the amplitide of the measured sidelobes with respect to the main beam.

\subsection{Source Design}

The source was excited with a Gunn oscillator from ZAX corporation model ZVTISC 6/1/145.1/.15 operating at $145 \mathrm{GHz}$, which fed a WR- 6 waveguide system. Table 1 shows the specifications of this oscillator. To stabilize the output power, its temperature was controlled with both a subminiature proportionally controlled heater (DN505-05) placed rigtt on the enclosure of the oscillator, and a $40 \mathrm{~W}$ heater controlled by an Omega CN7500 temperature controller heating an eluminum block that served as a thermal buffer. The system temperature was set to $36^{\circ} \mathrm{C}$ and continuously monitored showing a maximum steady-state drift of $2^{\circ} \mathrm{C}$. At the output of the oscillator, a variable attenuator was added to control the output signal and reduce the generation of standing waves.

To produce a wide angle antenna pattern, we used an open waveguide as a feed, which should in principie have a FWHM of nearly $90 \mathrm{deg}^{10}$ At the time we had no means to modify the waveguide used as a feed, so we were forced to use a regular waveguide section ending in a circular flange. This caused some distortions of the radiation pattern, as will be shown below.

The signal was chopped using a Stanford Research* SR540 optical chopper placed in front of the waveguide. It consists of an anodized aluminum wheel with 5 blades that rotate at a stable angular speed. We set the chopping frequency to be $5 \mathrm{~Hz}$, with a stability of $250 \mathrm{ppm} /{ }^{\circ} \mathrm{C}$ given by the manufacturer specifications.

The whole system was placed inside an aluminum box for protection, for thermal stability and for collimating the source beam in the forward direction. The box had an $7 \mathrm{~cm}$ window opening for the feed. The entire area surrounding the feed inside the box was covered with ECCOSORB' to avoid internal reflections, as can be seen in Figure 1.

\footnotetext{
"lttp://ww. thinksrs, con/

thttp://ww. eccosorb.con
}

\begin{tabular}{|l|c|c|c|c|c|}
\hline Model & $\begin{array}{c}\text { Frequency } \\
{[\mathrm{GHz}]}\end{array}$ & $\begin{array}{c}\text { Waveguide } \\
\text { Flange }\end{array}$ & Waveguide & $\begin{array}{c}\text { Power } \\
{[\mathrm{mW}]}\end{array}$ & $\begin{array}{c}\text { Tunning } \\
\text { Range }[\mathrm{GH}]\end{array}$ \\
\hline \hline ZVTISC6/1/145.1/.15 & 145.1 & ULG-387/UM & WR-6 & 1 & 0.15 \\
\hline
\end{tabular}

Table 1: ZAX, Gunn oscillator asembly specifications, ww. millimeterwave.com 


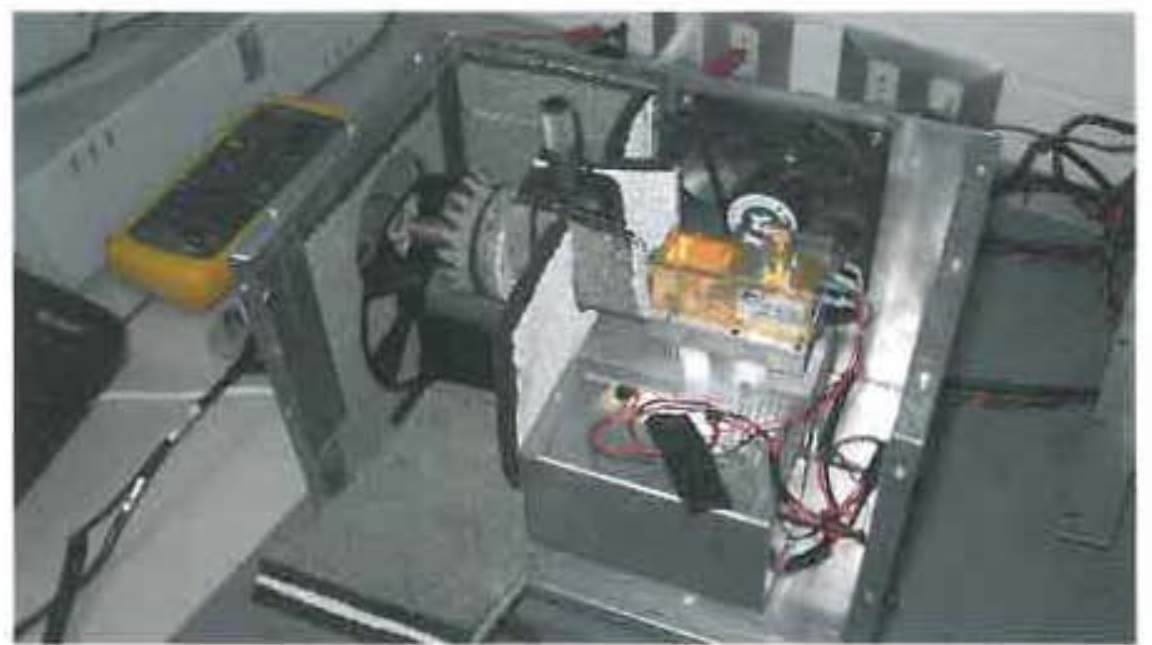

Figure 1: Microwave source interior setup. The feed shown in the figure was later replaced by an open waveguide with circular flange. The feed and surroundings are all covered with ECCOSORB to reduce reflexions. The fan was used to control temperature in case of overheating.

\subsection{Source Characterization}

The source's antenna pattern, output power and stability had to be characterized to help interpret the experimental results.

The antenna pattern of the source was measured using a Pacific Millimeter ${ }^{-1}$ broadband receiver diode (Mode! DD) with gain $G_{d}=900 \mathrm{mV} / \mathrm{mW}$, coupled to a rectangular pyramidal horn antenns of effective area $A_{r z}=$ $1.536 \mathrm{~cm}^{2}$. The signal was amplified with an instrumentation amplifier with gain $G=443$ and read using a NI-006 acquisition card. This receiver was mounted on a six-axis robot (ABB IRB1600), which allowed us to accurately place it at the desirec positions and orientations in front of the source with a position repeatability of $50 \mu \mathrm{m}$. Figure 2 shows this experimental setup. We sampled the source's profile along the $\mathrm{E}$ and $\mathrm{H}$ planes and along diagonals, making sure to keep the polarization angle to maximize the signal. The surveys were done at 20 and $30 \mathrm{~cm}$ from the source. To avoid having to deconvolve the measurements with the receiver pattern, we kept the receiver always pointing directly to the source feed and aligned with the polarization angle, so that all samples have the same receiver gain.

The results for the $\mathrm{E}$ and $\mathrm{H}$ planes are shown in figure 3 . The $\mathrm{H}$-plane profile presents a beam width at half power of $40 \mathrm{deg}$ and small features on the sides. The E-plane profile is more complicated presenting two strong sidelobes of intensity comparable to the central beam, which has a width of $20 \mathrm{deg}$. Both profiles stay stable when varying the distance to the receiver and the relative orientation of the experimental setup. One sidelobe in the E-plane, the one centered at $\theta=-30 \mathrm{deg}$, shows an unexpected oscillatory pattern which do not change when the robot setup is rotated, making us believe that it is real and caused by interference from within the source. The central intensity was unstable for both measurements, probably due to a standing wave formed between source and receiver horn.

These messurements were contrasted with numerical simulations of the waveguide feed done using the commercial software HFSS. We simulated an open waveguide with a circular flange. The computer aided design and simulation results for the model are shown in Figure 4. The results are in agreement to the laboratory measurements, showing that the large sidelobes in the E-plane are due to standing currents formed on the surface of the flange of the feed. ${ }^{11}$ In principle, this artifact can be mitigated and the feed pattern symmetrized by loading the flange with a layer of absorbing material. ${ }^{12}$

\footnotetext{
thttp://ww.pacificmillimeter.cou/

5http://vwr, ansys.com/
} 


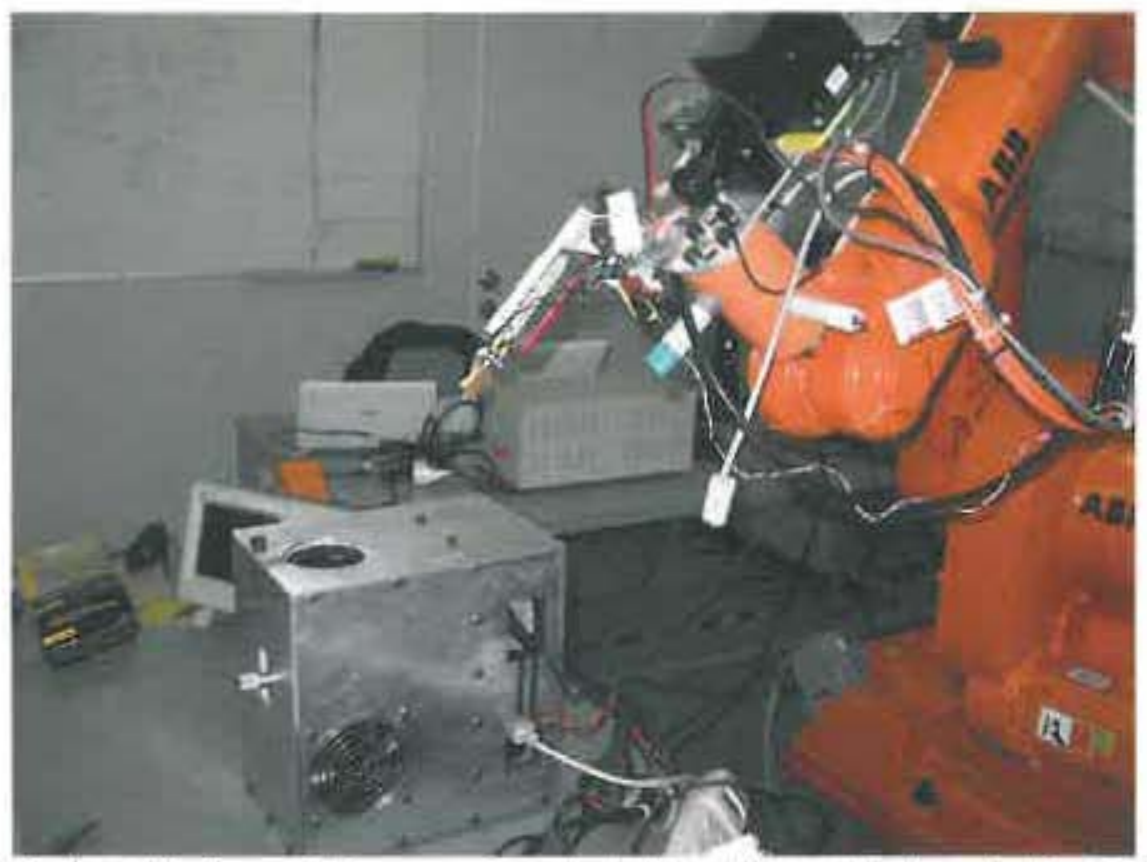

Figure 2: Mechanical positioning used in source charecterization. The golden horn is the receiver, being positioned by ABB IRB1600. In this picture, $\phi=180 \mathrm{deg}, \theta=-40 \mathrm{deg}$. To reduce reflexions from the robot, a square sheet of ECCOSORB was placed right behind the feed horn, which is not shown in this picture. Here the robot is sampling the E-plane of the source.

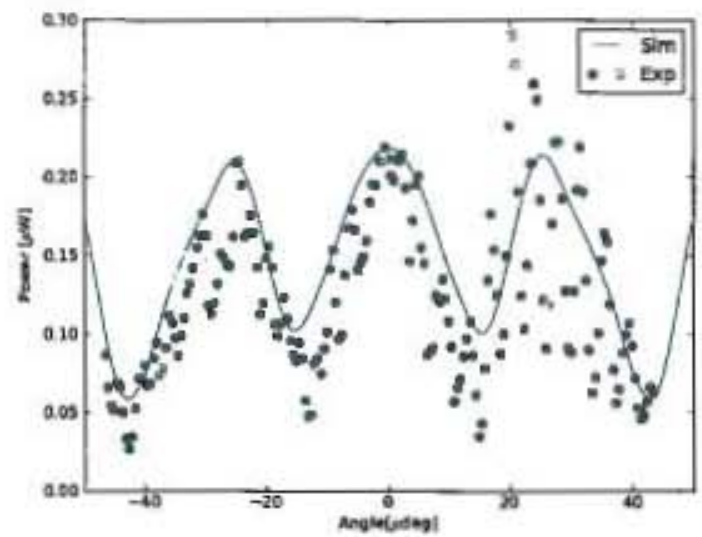

(a) E-plane

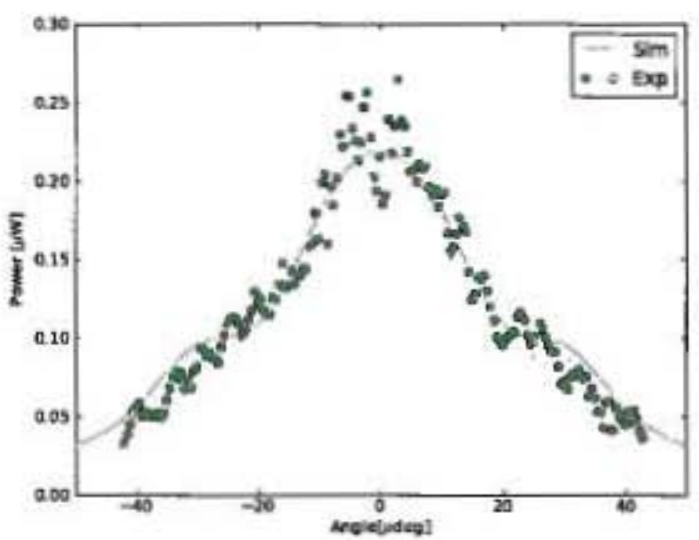

(b) H-plane

Figure 3: Measurement results for the $145 \mathrm{GHz}$ source sampling the $\mathrm{E}$ and $\mathrm{H}$-planes. The greed dots show the sampies taken $30 \mathrm{~cm}$ awsy from the source. These values can be converted into units of power per solid angle considering that the horn presented a solid angle of $1.7 \mathrm{msr}$ with respect to the source at this distance. The solid blue line shows the best fit of the simulated power pattern done on HFSS.

\subsection{Experimental Setup}

The final measurements were done in January 2011, right after the end of the observing season and before MBAC was definitively removed from the telescope. The source was mounted on top of the external ground shield, $13 \mathrm{~m}$ away from the primary mirror, as can be soen in Figure 5. A laser pointer mounted on the source wss used to aim it directly to the center of the primary mirror. After performing the standard biasing procedure on the camera, 


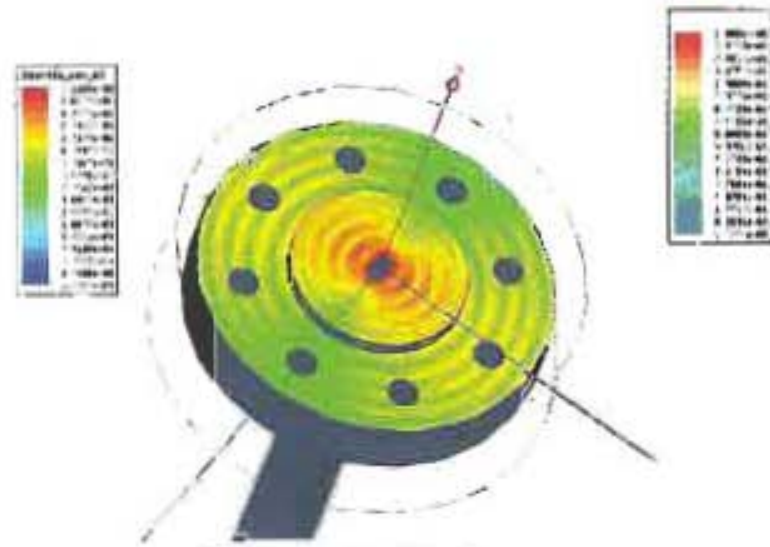

(a) Waveguide Model

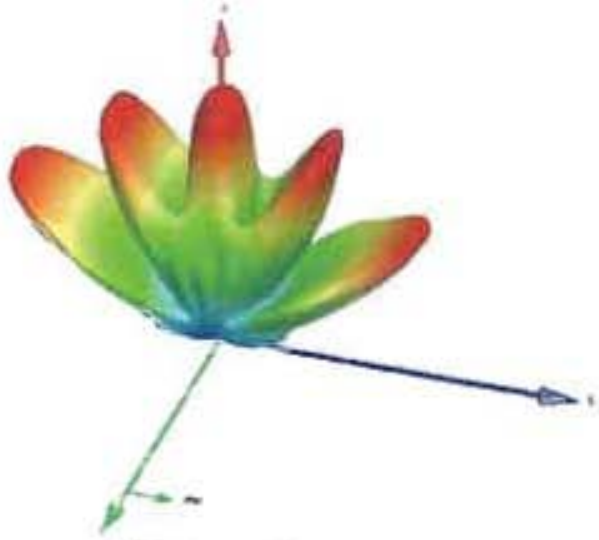

(b) Antenna Psttern

Figure 4: Sirmulated waveguide using HFSS. Figure (a) shows a model of the flange at the end of the feed. The currents on the surface are colored and denote the formetion of standing waves along the E-plane. Figure (b) shows the antenna power pattern of the waveguide feed. The large sidelobes on the E-plane are due to the currents on the surface of the flange.

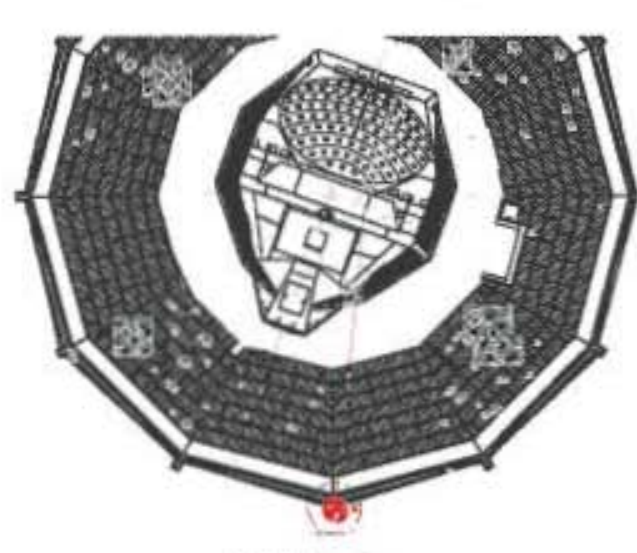

(a) Top view

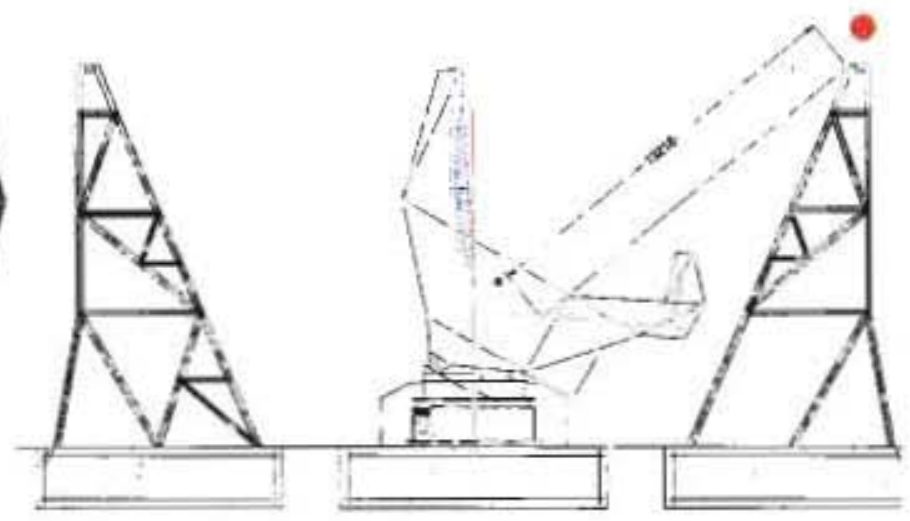

(b) Side view

Figure 5: Diagram with the location of the microwave source with respect to the telescope when mounted on the external ground shield. The distance to the center of the telescope is $13.2 \mathrm{~m}$. Figure (a) shows a top view of the configuration. The blue rectangle shows the position of the ladder in front of the primary mirror. The red lines trace the rays that may bounce on the ladder and go into the camera. At the angle at which this happens we detect a strong sidelobe as shown in Figure 8 , so we believe that the sidelobe was cause by the ladder. The ladder is not there during normal observations. Figure (b) shows the side view of the configuration. Here the telescope is pointing to its lowest possible elevation (31 deg), existing a direct line of sight from the source to the window of the camera. This means that at this position we are directly measuring the sidelobe rejection of the camere.

we scanned the telescope for the full range in azimuth ( 0 - $360 \mathrm{deg})$ at constant elevation for six elevation settings ranging between $31 \mathrm{deg}$ and $60 \mathrm{deg}$ with steps of $5 \mathrm{deg}$, with a scan speed of $\Omega=0.3 \mathrm{deg} / \mathrm{s}$. The signal captured in NIBAC was stored in the standard time order datastream ('TOD) format used for sstronomical observations for its analysis. The temperature of the source was monitored during the experiment, showing variations with a standard deviation of $1.8^{\circ} \mathrm{C}$. 


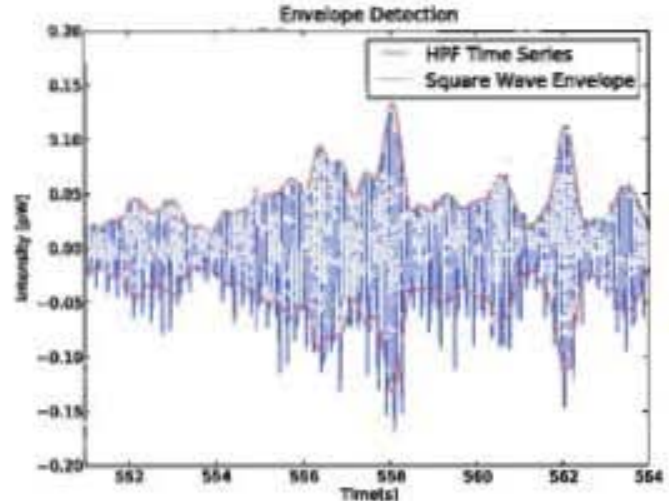

(a)

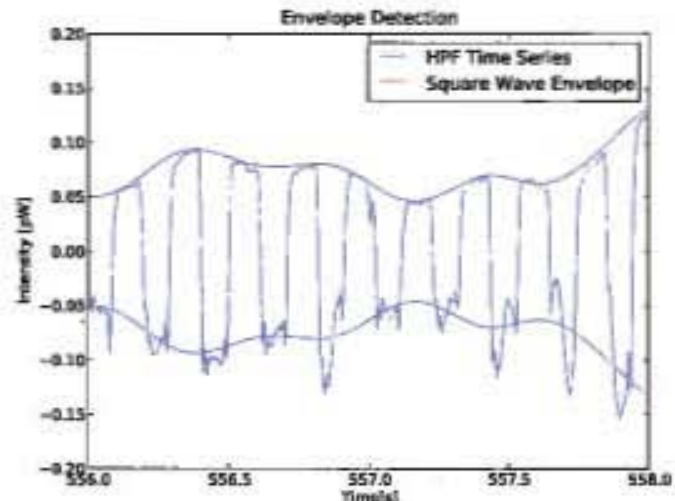

(b)

Figure 6: Envelope detection performance. Note that the estimated envelope follows the amplitude, however high frequencies are not followed.

\section{DATA ANALYSIS}

We are interested in measuring the amplitude of the chopped signal as a function of telescope orientation. Given that the telescope was moving while the data was recorded, each chop had a different orientation. We used a time domain Hilbert transform method to extract the envelope of the chopped signal. ${ }^{13}$

Let $C(t)$ be a square wave carrier signal of period $T$, and env $(t)$ a slow varying function in time, then the square wuve modulated by the envelope can be expressed as

$$
s(t)=C(t) \times e n v^{\prime}(t)=\left(1+\frac{4}{\pi} \sum_{n=1,3,5 \ldots}^{\infty} \frac{1}{n} \sin \frac{2 \pi n t}{T}\right) \times e n v(t) .
$$

Band-pass filtering the above expression to isolate the first harmonic, we obtain

$$
s_{1}(t)=\frac{4}{\pi} \epsilon n v(t) \sin \frac{2 \pi t}{T} .
$$

Provided that the carrier frequency $f=1 / T=5 \mathrm{~Hz}$ is above the frequency content of env(t), we can use the Becirosian identity ${ }^{14}$ to show that the Hilbert transform of $s_{1}(t)$ is

$$
H\left[s_{1}\right]=-\frac{4}{\pi} \operatorname{env}(t) \cos \frac{2 \pi t}{T},
$$

so we can rocover the envelope by writing

$$
\operatorname{env}(t)=\frac{\pi}{4} \sqrt{s_{1}(t)^{2}+H\left[s_{1}(t)\right]^{2}} .
$$

The bandwidth of the envelope is ultimately determined by the band-pass filter, setting the maximum resolution at which we can recover the sidelobes. For noisy data, increasing the bandwidth may also decrease the signal to noise ratio. To find the sweet spot, we performed a simulation for a slow varying signal plus noise as measured from the data. We found that a $2 \mathrm{~Hz}$ bandwidth maximizes the signal to noise, leaving a $3 \%$ error in the envelope power.

Figure 6 shows the chopped signal in good agreement with the envelope recovered by our method.

Out of 1024 detectors in the focal plase, 633 were correctly biased and could be used to collect.data during the experiment, giving a fair sample of the distribution of the signal across the array. Figure 7 provides a map 


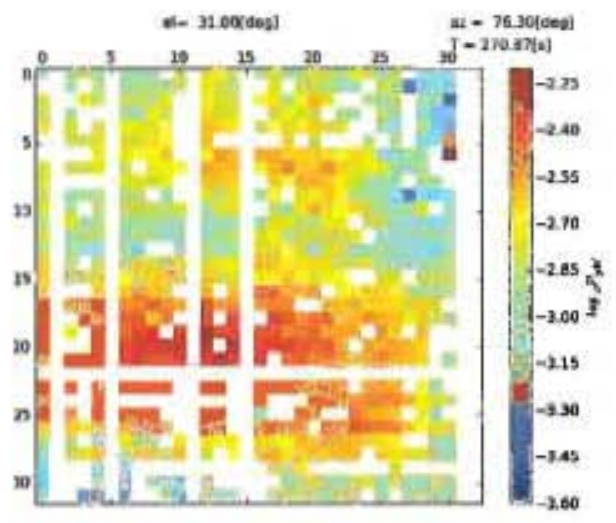

Figure 7: Instantaneous distribution of the signal acroes the detector array when pointing towards $\dot{\phi}_{a z}=76.3 \mathrm{deg}$ and $\theta_{e l}=31 \mathrm{deg}$. We believe that the non-homogeneous illumination is produced by interference patterns produced when a colerent source reaches the focal plane from two or more paths.

of the signal across the focal plane by comparing the response of different detectors at a short azimuth range of $1 \mathrm{deg}$ when pointing towards $\phi_{a z}=76.3 \mathrm{deg}$ and $\theta_{e l}=31 \mathrm{deg}$. The signal is clearly non-homogenous, forming moring stripes across the array as the telescope scans. Given that the source is coherent and that it is placed near the telescope, we think that they ure some sort of interference patterns due to the presence of multiple optical paths and do not represent proper sidelobes, which are expected to be much wider. For this reason, we estimated the signal at every point as the average signal over all detectors.

Given the proximity of the source, different parts of the telescope are illuminated with different incidence angles and the far field condition is not met. Despite this, given that the sidelobes are produced by diffraction from the telescope structure, and given that those structures are in the far field of the camera alone, we expect that the response of the telescope would not differ much from its response to a source placed in the far field. In other words, the proximity of the source will only distort the angular distribution of the far sidelobes but not their overedl intensity.

To estimate the attenuation of the sidelobes with respect to the central beam, we need to estimate the power that would be received by the telescope if the source were placed st the far field and applied the same power flux per unit area as the source in the near field. In our laboratory tests we measured the power output of the source with the same attenuation as the one used in the experiment at the site. Fitting the simulated antenna pattern to the laboratory measurements, we could estimate the power radiated in the forward direction as a function of output solid angle. Then we considered that the effective aperture of the telescope, as measured from planet observations, is $A_{e}=18.6 \pm 0.1 \mathrm{~m}^{2}$, and that the distance of the source to the center of the telescope was $13.2 \mathrm{~m}$, to estimate that the angular size of the aperture in front of the source would roughly be 21 deg. Integrating the source power pattern over that angle, we obtain the equivalent power of the source seen in the main beam. This power still needs to be attenuated by the power efficiency of the telescope, which is $\eta_{148 G H z}=0.16$ as measured from planet observations, yielding $P_{0}=1.40 \pm 0.13 \mu \mathrm{W}$. The uncertainty here represents the error from the laboratory measurements, although it does not consider the uncertainty in the calibration of the ciode and potential changes in the power delivered by the Gunn source as a function of operating temperature at the site. We conservatively estimate that the results are accurate within $5 \mathrm{~dB}$.

Referencing to this value, our results are provided in decibels as

$$
P_{d B}=10 \log _{10} \frac{P_{b l}}{P_{0}},
$$

where $P_{s t}$ is the measured sidelobe power in Watts. 


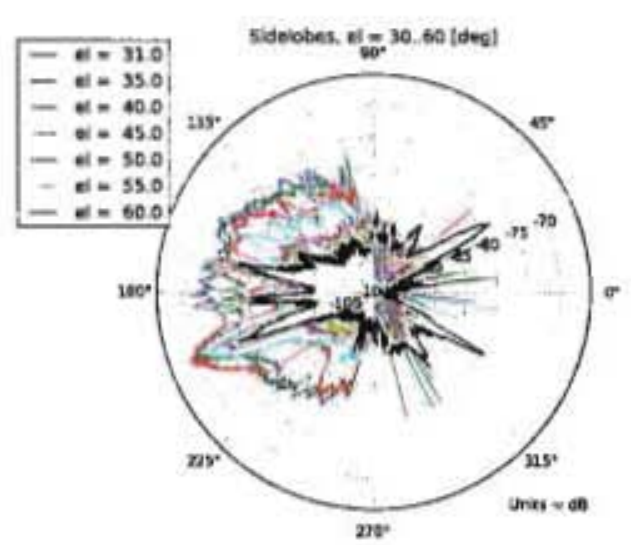

(a) Test 1

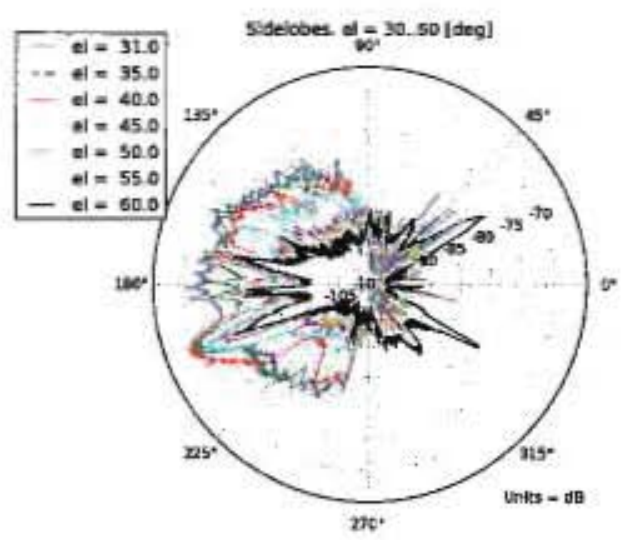

(b) Test 2

Figure 8: Azimuthal distribution of the far sidelobe power measured in two repetitions of the experiment (a. and b). The units are in decibels with respect to the expected central power $P_{0}=1.40 \pm 0.13 \mu \mathrm{W}$. The two experiments are consistent with each other. The strong sidelobe at $a z=200 \mathrm{deg}$ is explained by a ladder that was placed in front of the primary mirror during the experiment.

\section{RESULTS AND DISCUSSION}

Figure 8 shows the distribution of the signal in aximuth for different elevations. As expected, the intensity of the signal is generally higher for orientations closer to the central beam. The highest peak in intensity is found at $a x=200 \mathrm{deg}$ at an elevation of $40 \mathrm{deg}$. This angle is consistent with reflexions from a ladder that was circumstantially placed in front of the primary mirror for several servicing labors happening at the time, but that is not there during normal observations, as shown in Figure 5a. Disregarding this peak, the sidelobe contribution ranges between -95 and $-75 \mathrm{~dB}$.

The uncertainties are dominated by the estimation of the central power. Studying the variability of this estimation as a function of geometrical parameters we expect our result to be accurate within $5 \mathrm{~dB}$. Above the experimental uncertainties, our result demonstrates that the far sidelobe level of this telescope is beyond the expected value for on-axis telescopes, which are of order $-50 \mathrm{~dB} \cdot{ }^{9}$ This is anticipsted for the off-axis configuration in use and is necessary to achieve the science goals of such precision mapping experiments.

Using these results it is possible to estimate the amount of loading expected on the detectors coming from the ground outside the ground shield. This radiation diffracts over the edge of the ground shield and shines on the telescope from the same location as the source, but distributed along the entire circumference of the ground shield. Every differential solid angle projected by the ground to the edge of the ground shield produces a single ray that will get diffracted and attenuated before contributing to the total flux that reaches the telescope. Integrating these rays for the surface brightness of the ground at $300 \mathrm{~K}$, and applying Keller's diffraction formula for a wedge ${ }^{15}$ to calculate the sttemuation of each ray, we obtain that the flux power density per unit area at the center of the telescope would be $1.9 \mathrm{nW} / \mathrm{m}^{2}$. Mlultiplying this by the effective area of the telescope and attenuating by the average sidelobe response at each elevation, we obtain the total loading expected at the corresponding elevation. The results are shown in Figure 9. Considering that the total loading measured during nominal observations $\left(\theta_{e t}=51.5 \mathrm{deg}\right)$ is of order $2 \mathrm{pW}$, it is clear that the ground loading is a negligible contribution. The dominate component of the detector loading is due to the atmosphere and spillover loading from within the telescope."

\section{CONCLUSION}

A satisfactory measurement of the far sidelobes of the ACT telescope was presented. The technique uses a source located near the telescope and an understunding of the diffrected power coupling to the detector arrav to estimate the overall intensity of the sideiobes expected from a source located in the far field of the telescope. 


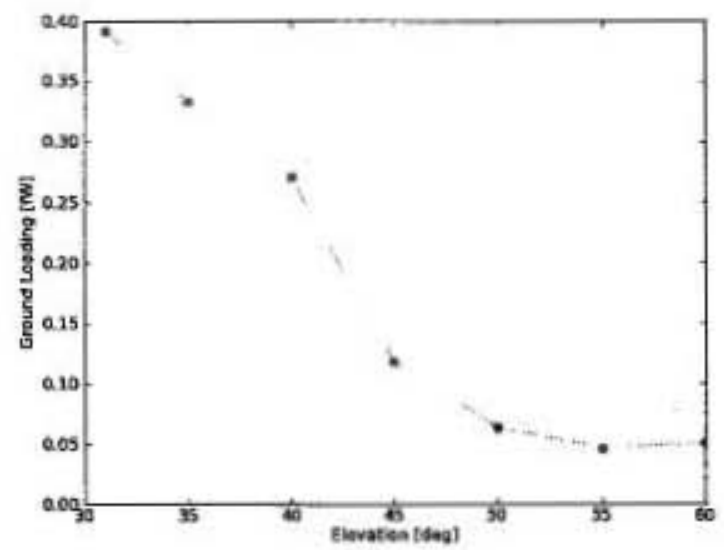

Flzure 9: Expected loading from the ground outside of the ground shield leaked in through the far sidelobes.

The far sidelobe level was measured to be attenuated $-75 \mathrm{~dB}$ with respect to the main beam of the telescope, which satisfies all the design expectations for the system, situating it quite above the performance of regular on-axis systems. This result, plus the effect of diffraction over the ground shield structure, imply that the ground contribute with less than $0.3 \mathrm{fW}$ of power, which is negligible compared to other sources of loading.

\section{ACKNOWLEDGMENTS}

This project was partially funded by a FONDECYT Iniciación N`11100147 award, a VRI Inicio 39/2010 award and FONDAP. We thank Lyman Page (Princeton University), for lending us the Gunn osclllator and chopper used, and for many suggestions and guiding. We also thank Juan Carlos Fluxa and Sergio Rojas for their collsboration in the project. We specially thank Masao Uehara for his help in performing the experiment at tàe site. We thank Parque Astronomico Atacama (CONICYT), in which the ACT telescope is located. We thank the Electrical Engineering department at PUC for letting us use the robotic laboratory to do the source characterization.

\section{REFERENCES}

[1] Fowler, J. W., Niemack, M. D., Dicker, S. R., Aboobaker, A. M., Ade, P. A. R., Battistelli, E. S., Devlin, M. J., Fisher, R. P., Halpern, M., Hargrave, P. C., Hincks, A. D., Kaul, M., Klein, J., Lau, J. M., Limon, M., Marriage, T. A., Mauskopf, P. D., Page, L., Staggs, S. T., Swetz, D. S, Switzer, E. R., Thornton, R. J., and Tucker, C. E., "Optical design of the Atacama Cosmology Telescope and the Millimeter Bolometric Array Camera," Applied Optics 46(17), 3444-3454 (2007).

[2] Swetz, D. S., Ade, P. A. R., Amiri, M., Appel, J. W., Battistelli, E. S., Burger, B., Chervenak, J., Devlin, M. J., Dicker, S. R., Doriese, W. B., Dünner, R., Essinger-Hileman, T., Fisher, R. P., Fowler, J. W., Halpern, M., Hasseifield, M., Hilton, G. C., Hincks, A. D., Irwin, K. D., Jarosik, N., Kaul, M., Klein, J., Lau, J. M., Limon, M., Marriage, T. A., Marsien, D., Martocci, K., Mauskopf, P., Moseley, H., Netterfield, C. B., Niemack, M. D., Nolta, M. R., Page, L. A., Parker, L., Staggs, S. T., Stryzak, O., Switzer, E. R., Thornton, R., Tucker, C., Wollack, E., and Zheo, Y., "Overview of the Atacama Cosmology Telescope: Recelver, Instrumentation, and Telescope Systems," Astrophysical Journal, Supplement 194, 41- + (June 2011).

[3] Sunysev, R. A. and Zel'dovich, Y. B., "The Spectrum of Primordial Radiation, its Distortions and their Significance," Comments on Astrophysics and Space Physics 2, 66 (Mar. 1970).

[4] Hincks, A. D., Acquaviva, V., Ade, P. A. R., Aguirre, P., Amiri, M., Appel, J. W., Barrientos, L. F., Battistelli, E. S., Bond, J. R., Brown, B., Burger, B., Chervenak, J., Das, S., Devlin, M. J., Dicker, S. R., Doriese, W. B., Dunkley, J., Dünner, R., Essinger-Hileman, T., Fisher, R. P., Fowler, J. W., Hajian, A., 
Halpern, M., Hasselfield, MI., Hernández-Monteagudo, C., Hilton, G. C., Hilton, M., Hlozek, R., Huffenberger, K. M., Hughes, D. H., Hughes, J. P., Infante, L., Irwin, K. D., Jimenez, R., Juin, J. B., Kaul, M., Klein, J., Kosowsky, A., Lau, J. M., Limon, M., Lin, Y.-T., Lupton, R. H., Marriage, T. A., Marsden, D., Martocci, K., Mauskopf, P., Menanteau, F., Moodley, K., Moseley, H., Netterfield, C. B., Niemack, M. D, Nolta, M. R., Page, L. A., Parker, L., Partridge, B., Quintana, H., Reid, B., Sehgal, N., Sievers, J., Spergel, D. N., Staggs, S. T., Stryzak, O, Swetz, D. S., Switzer, E. R., Thornton, R., Trac, H., Tucker, C., Verde, L., Warne, R., Wilson, G., Wollack, E., and Zhao, Y., "The Atacama Cosmology Telescope (ACT): Beam Profiles and First SZ Cluster Maps," Astrophysical Journal, Supplement 191, 423-438 (Dec. 2010).

[5] Marriage, T. A., Acquaviva, V., Ade, P. A. R., Aguirre, P., Amiri, M., Appel, J. W., Barrientos, L. F., Battistelli, E. S., Bond, J. R., Brown, B., Burger, B., Cherverak, J., Das, S., Devlin, M. J., Dicker, S. R., Bertrand Doriese, W., Dunkley, J., Dünner, R., Essinger-Hileman, T., Fisher, R. P., Fowler, J. W., Hajian, A., Halpern, Mi., Hasselfield, M., Hernández-Monteagudo, C., Hilton, G. C., Hilton, M., Hincks, A. D., Hlozek, R., Huffenberger, K. M., Handel Hughes, D., Hughes, J. P., Infante, L., Irwin, K. D., Bsptiste Juin, J., Kaul, M., Klein, J., Kosowsky, A., Lau, J. M., Limon, M., Lin, Y.-T., Lupton, R. H., Marsden, D., Martocci, K., Mauskopf, P., Menanteau, F., Moodley, K., Moseley, H., Netterfield, C. B., Niemack, M. D., Nolta, M. R., Page, L. A., Parker, L., Partridge, B., Quintana, H., Reese, E. D., Reid, B., Sehgal, N., Sherwin, B. D., Sievers, J., Spergel, D. N., Staggs, S. T., Swetz, D. S., Switzer, E. R., Thornton, R., Trac, H., Tucker, C., Warne, R., Wilson, G., Wollack, E., and Zhao, Y., -The Atacama Cosmology Telescope: Sunyaev-Zei'dovich-Selected Galaxy Clusters at $148 \mathrm{GHz}$ in the 2008 Survey?' Astrophysical Journal 737, 61- (Aug. 2011).

[6] Fowier, J. W., Acquaviva, V., Ade, P. A. R., Aguirre, P., Amiri, M., Appel, J. W., Barrientos, L. F., Battistelli, E. S., Bond, J. R., Brown, B., Burger, B., Chervenak, J., Das, S., Devlin, M. J., Dicker, S. R., Doriese, W. B., Dunkley, J., Dünner, R., Essinger-Hileman, T., Fisher, R. P., Hajian, A., Halpern, M., Hasselfield, M., Hernández-Monteagudo, C., Hilton, G. C., Hilton, M., Hincks, A. D., Hlozek, R., Huffenberget, K. M., Hughes, D. H., Hughes, J. P., Infante, L., Irwin, K. D., Jimenez, R., Juin, J. B., Kaul, M., Klein, J., Kosowsky, A., Lau, J. M., Limon, M., Lin, Y., Lupton, R. H., Marriage, T. A., Marsden, D., Martocci, K., Mauskopf, P., Menanteau, F., Moodley, K., Moseley, H., Netterfield, C. B., Niemack, M. D., Nolta, M. R., Page, L. A., Parker, L., Partridge, B., Quintana, H., Reid, B., Sehgal, N., Sievers, J., Spergel, D. N., Staggs, S. T., Swetz, D. S., Switzer, E. R., Thornton, R., Trac, H., Tucker, C., Verde, L., Warne, R., Wilson, G., Wollack, E., and Zhao, Y., "The Atacama Cosmology Telescope: A Measurement of the $600<\ell<8000$ Cosmic Nicrowave Background Power Spectrum at $148 \mathrm{GHz}$," Astrophysical Journal 722, 1148-1161 (Oct. 2010).

[7] Das, S., Marriage, T. A., Ade, P. A. R., Aguirre, P., Amiri, M., Appel, J. W., Barriento6, L. F., Battistelli, E. S., Bond, J. R., Brown, B., Burger, B., Chervenak, J., Devlin, M. J., Dicker, S. R., Bertrand Doriese, W., Dunkley, J., Dünner, R., Essinger-Hileman, T., Fisher, R. P., Fowler, J. W., Hajian, A., Halpern, M., Hasselfield, M., Hernándea-Monteagudo, C., Hilton, G. C., Hilton, M., Hincks, A. D., Hlozek, R., Huffenberger, K. M., Hughes, D. H., Hughes, J. P., Infante, L., Irwin, K. D., Baptiste Juin, J., Kaul, M., Klein, J., Kosowsiky, A., Lau, J. M., Limon, M., Lin, Y.-T., Lupton, R. H., Marsden, D., Martocci, K., Mauskopf, P., Menanteau, F., Moodley, K., Moseley, H., Netterfield, C. B., Nlemack, M. D., Nolta, M. R., Page, L. A., Parker, L., Partridge, B., Reid, B., Sehgal, N., Sherwin, B. D., Sievers, J., Spergel, D. N., Staggs, S. T., Swetz, D. S., Switzer, E. R., Thornton, R., Trac, H., Tucker, C., Warne, R., Wollack, E., and Zhso, Y., "The Atacama Cosmology Telescope: A Measurement of the Cosmic Mlicrowave Background Power Spectrum at 148 and $218 \mathrm{GHz}$ from the 2008 Southern Survey," Astrophysical Journal 729, 62-+ (Mar. 2011).

[8] Dunkley, J., Hlozek, R., Sievers, J., Acquaviva, V., Ade, P. A. R., Aguirre, P., Amiri, M., Appel, J. W., Barrientos, L. F., Battistelli, E. S., Bond, J. R., Brown, B., Burger, B., Chervenak, J., Das, S., Devlin, M. J., Dicker, S. R., Bertrand Doriese, W., Dünner, R., Essinger-Hileman, T., Fisher, R. P., Fowler, J. W., Hajian, A., Halpern, M., Hasselfield, M., Hernández-Monteagudo, C., Hilton, G. C., Hilton, M., Hincks, A. D., Huffenberger, K. M., Hughes, D. H., Hughes, J. P., Infante, L., Irwin, K. D., Juin, J. B., Kaul, M., Klein, J., Kosowsky, A., Lau, J. M., Limon, M., Lin, Y.-T., Lupton, R. H., Marriage, T. A., Marsden, D., Mauskopf, P., Menanteau, F., Moodley, K., Moseley, H., Netterfield, C. B., Niemack, M. D., Nolta, M. R., Page, L. A., Parker, L., Partridge, B., Reid, B., Sehgal, N., Sherwin, B., Spergel, D. N., Staggs, S. T., Swetz, 
D. S., Switzer, E. R., Thornton, R., Trac, H., Tucker, C., Warne, R., Wollack, E., and Zheo, Y., "The Atacama Cosmology Telescope: Cosmological Parameters from the 2008 Power Spectrum," Astrophysical Journal 739, 52-+ (Sept. 2011).

[9] Lockman, F. J., "Stray Radiation: Causes, Curses, and Cures," in [Single-Dish Radio Astronomy: Techniques and Applications], S. Stanimirovic, D. Altschuler, P. Goldsmith, \& C. Salter, ed., Astronomical Society of the Pacific Conference Series 278, 397-411 (Dec. 2002).

[10] Silver, S., [Microwave Antenna Theory and Design], Iee Electromagnetic Waves Series, P. Peregrinus (1949).

[11] James, G. and of Electrical Engineers, L., [Geometrical Theory of Diffrection for Electromagnetic Waves], Iee Electromagnetic Waves Series, P. Peregrinus (1986).

[12] Yoshitomi, K. and Sharobim, H. R., 'Radiation from a rectangular waveguide with a lossy flange," IEEE Thansactions on Antennas and Propagation 42, 1398-1403 (Oct. 1994).

[13] Feldman, M., "Hilbert transform in vibration annlysis," Mechanical Systems and Signal Processing 25, 735-802 (Apr. 2011).

[14] Bedrosian, E., "A product theorem for hilbert transforms," Rand Corporation Memorandum RM-3439-PR (1962).

[15] McNamara, D., Pistorius, C., and Malherbe, J., [Introduction to the Uniform Geometrical Theory of Diffraction!, Microwave Library, Artech House (1990). 